\title{
A space science boom or death by a thousand small satellites
}

\author{
Small satellites are revolutionizing the way we access space for commerce and science, including astronomy and \\ planetary science. Yet, like many breakthrough technologies, it has a double-edge.
}

$\mathrm{P}$ icture this: a farmer in Burundi uses their smartphone to access the internet to learn about next week's weather forecast, a group of university students plan space observations of the Andromeda galaxy, and a government agency sees a tsunami-like structure forming in the middle of the Pacific Ocean in real time and alerts emergency services. Now picture this: a mother is showing her son the night sky when they notice a bright trail running through the Ursa Major, two objects crash around 1,000 kilometres above the Earth initiating a cascade of catastrophic collisions, and a ground-based telescope follows up the latest gravitational wave signal only to detect a very bright light crossing its field of view. What is the common thread in these situations? Small satellites. This issue's Focus explores the relevance of small satellites to astronomy and planetary science, following a meeting on this topic hosted at the Nature London offices in November 2019, which brought together astronomers, industry experts and policy makers.

According to the latest numbers, there are currently around 3,000 artificial satellites orbiting Earth, roughly 2,000 of which are in low-Earth orbit (up to altitudes of $2,000 \mathrm{~km}$, but typically below $1,000 \mathrm{~km}$ ). If commercial satellite operators continue to have their way, this number is set to increase by orders of magnitude within the next decade. Indeed, we have entered the era of 'New Space', typically defined as the recent commercialization of the space sector'. Simply put, the exploration (and exploitation) of space is no longer the sole remit of government agencies. Instead, maturing and emerging technologies (and new business models) have allowed private companies to produce and launch satellites cheaply and quickly.

This revolution is most visible in the arena of small satellites and cube satellites. Weighing no more than $500 \mathrm{~kg}$ and offering standardized methods of payload integration and launching capabilities, these platforms (the whole supporting structure within which the payload is hosted) can be produced in bulk (several a day) for a fraction of the cost of traditional bulkier satellites. Importantly, a standardized platform means that off-the-shelf solutions for both the platform itself and its payload are possible (see the Perspectives of Doug Liddle et al. and Philip Allen et al.). The threshold for accessing space has plummeted; for example, consider that the launch cost per kilogram has gone down by almost two orders of magnitude within the last 40 years. The opportunities are countless, for commerce, research and education. As is often the case, commercial endeavours lead the pack, with several major players focusing on large satellite 'constellations'. One of their main selling points is that these constellations will offer a gamut of services (such as broadband internet) to far-flung places on Earth. Yet this veritable 'democratization' of space does not come without its own issues.

Recently, images of the night sky dotted with satellite trains making their way from Earth to their low-Earth orbits have caused outrage. Beyond their launch, depending on the altitude of their final orbit, these satellites also reflect sunlight and imprint themselves as trails in the observations of ground-based telescopes around the globe. They are furthermore sources of radio frequency interference, affecting radio telescopes. The problem is already so serious, and set to become worse, that several astronomy-related professional bodies, including the International Astronomical Union, the American Astronomical Society and the European Astronomical society have issued stark warnings about the impact these satellite constellations will pose to current and next-generation ground-based observatories (see the Comments by Robert Massey et al. and Igor Levchenko et al.).

Moreover, according to DISCOS (Database and Information System Characterising Objects in Space), up to 40,000 unidentified artificial objects bigger than $10 \mathrm{~cm}$ are currently in near-Earth orbits. As the most recent alarm of an imminent collision between the defunct Kosmos-2004 Russian satellite and the Chinese Chang Zheng rocket stage showed, the ever-more-densely populated low-Earth orbits increase the danger of debris multiplying through collisions, eventually jeopardizing the operation of functional satellites.

Not all is doom and gloom though. There is a vast scientific potential in these relatively new tools to access space, as detailed in the Perspective by Stephen Serjeant et al. Several major space-faring nations are currently exploring the use of small satellites for research (for example, NASA, ESA and CASC) and there are already many successful examples of small satellites performing high-quality research (for example, the Sino-Italian PolarLight and the most recent NASA HaloSat results by Philip Kaaret et al.). The lower price for entry also allows smaller players to claim a stake in space astronomy (as discussed in the Comment by Richard Archer et al. and this issue's Mission Control) or use mini space observatories for education and outreach purposes, like ESA's Fly Your Satellite! programme or the US Department of Education's CTE Mission: CubeSat programme.

As with any tool, it is up to us to decide how best to use it. Our community is rightfully alarmed by the unchecked proliferation of small satellites around Earth and their impact on the way we do our science or, even more fundamentally, on our universal right to an unaltered night sky (as discussed in the Perspective by Aparna Venkatesan et al.). Astronomers need to be staunch advocates for accountability in the private space sector and a globally coordinated approach to space regulation. At the same time, however, we must not lose sight of the technology, research and education opportunities offered by small satellites (explored in James Endicott's Comment) and their true potential to equalize access to space (see the Comment by Luca Maresi and Alessandro Zuccaro Marchi). It's a brave new world for space astronomy and we must ensure that commercial interests do not edge out scientific interests or spoil the night sky or near-Earth space for generations to come.

Published online: 6 November 2020 https://doi.org/10.1038/s41550-020-01257-0 